\title{
DETERMINAÇÃO COLORIMÉTRICA DO COBRE EM FERTILIZANTES *
}

\author{
J. C. Alcarde \\ R. A. Catani \\ C. O. PONCHIO
}

\begin{abstract}
O presente trabalho descreve os estudos efetuados com o objetivo de adaptar o método colorimétrico do dietilditiocarbamato à determinação do cobre em fertilizantes. Foram estudadas a influência do ânion fosfato no citado método, processos de preparo dos extratos de fertilizantes contendo resíduos orgânicos, assim como foram avaliadas sua precisão e exatidão, comparativamente com o método iodométrico.

Os resultados permitiram concluir que o método colorimétrico do dietilditiocarbamato não é afetado por uma quantidade de fosfato de até $4 \mathrm{mg}$. Apresenta também precisão e exatidão adequadas para a determinação do cobre em fertilizantes, comparáveis às do método iodométrico, associadas ainda a sua simplicidade e sensibilidade.
\end{abstract}

\section{INTRODUÇÃO}

Atualmente está se tornando rotineira a necessidade de correção de deficiencias de micronutrientes nas culturas em geral. Embora essa correção possa ser feita pela aplicação isolada de materiais que contém os referidos nutrientes, quer no solo como em pulverização foliar, é recomendado também o uso de misturas de fertilizantes contendo micronutrientes. Tal prática apresenta as vantagens de distribuir mais uniformemente os micronutrientes e de reduzir o número de aplicação relativas a adubação (NELSON \& TERMAN' 1963).

Entre os micronutrientes que são incorporados às misturas de fertilizantes encontra-se o cobre.

A determinação do cobre nos fertilizantes tem sido feita volumetricamente, por iodometria, ou espectrofotometricamente, por absorção atomica (OFFICIAL METHODS OF ANALYSIS OF THE A. O. A. C. 1965). O método iodométrico, embora relativamente simples,

\footnotetext{
* Entregue para publicação em 21-12-1971.

** Departamento de Química ESALQ.

*:* Bolsista do CNPq.
} 
é de sensibilidade limitada. O método baseado na fotometria de chama de absorção, apesar de rápido e sensível, demanda o emprego de aparelhos de custo nem sempre acessível.

Em face disso procurou-se adaptar um método colorimétrico para a presente determinação. $O$ método do dietilditiocarbamato de sódio (SANDELL, 1959) mostrou possuir qualidades que recomendassem o seu estudo. O citado método tem sido aplicado na determinação do cobre em solos e plantas (CHENG \& BRAY, 1953; JACKSON, 1958: FISKELL, 1965) e foi estudado em seus diversos aspectos por JACINTHO (1967).

O presente trabalho constituiu-se no estudo do referido método colorimétrico com o objetivo de aplicá-lo na determinação do cobre em fertilizantes. Assim, foram abordados o efeito da presença de elevadas concentrações do ânion fosfato, processos de preparo dos extratos de fertilizantes minerais e de fertilizantes contendo resíduos organicos e finalmente o método foi comparado com o iodométrico, avaliando-se sua precisão e exatidão.

\section{MATERIAL E MÉTODOS}

\section{Material}

O material constou de 5 misturas de fertilizantes, identificadas como A, B, C, D e E, nas quais foram adicionadas quantidades variáveis de cobre. As misturas A e B foram preparadas a partir de fertilizantes simples comerciais e a composição porcentual das mesmas está descrita no quadro 1.

A mistura A contém, aproximadamente, $6,0 \%$ de $\mathrm{N}, 15 \%$ de $\mathrm{P}_{2} 0_{5}$ e $12 \%$ de $\mathrm{K}_{2} 0$ e a mistura $\mathrm{B}, 8,5 \%$ de $\mathrm{N}, 15 \%$ de $\mathrm{P}_{2} \mathrm{O}_{5}$ e $12 \%$ de $\mathrm{K}_{2} 0$.

Quadro 1 - Composição porcentual das misturas de fertilizantes A e B, preparadas em laboratório.

\begin{tabular}{l|cc}
\hline \multirow{2}{*}{ Componentes } & \multicolumn{2}{c}{ Misturas } \\
\cline { 2 - 3 } & A & B \\
\hline Superfosfato simples & 30 & 30 \\
Superfosfato triplo & 20 & 20 \\
Cloreto de potássio & 20 & 20 \\
Salitre do Chile & 10 & - \\
Uréia & - & 10 \\
Sulfato de amônio & 20 & 20 \\
\hline
\end{tabular}


As misturas $\mathrm{C}, \mathrm{D}$ e $\mathrm{E}$ foram produtos comerciais com a seguinte composição: mistura $C(5,0-15,0-9,0)$, mistura D $(12,0-8,0-$ 7,0 , contendo $0,1 \%$ de zinco e $0,05 \%$ de boro) e mistura $\mathrm{E}$ ( $12,0-$ $6,0-10,0$, contendo $0,1 \%$ de zinco e $0,05 \%$ de boro).

Em cada mistura foram adicionadas quantidades variáveis de $\mathrm{CuSO}_{4} .5 \mathrm{H}_{2} \mathrm{O}$ p.a, de maneira a obter amostras contendo, aproximadamente, $2 \%, 4 \%$ e $8 \%$ daquele sal, correspondendo, aproximadamente, a $0,5 \%, 1 \%$ e $2 \%$ de cobre. O teor mínimo de $0,5 \%$ de cobre foi adotado em face do que menciona NELSON \& TERMAN (1963).

\section{Reativos} guintes :

Dentre os reativos utilizados merecem ser mencionados os se-

Solução de hidróxido de amônio $(1+1)$ destilado.

Solução de HC1 $(1+1)$ destilado.

Solução de HC1 0,5 $\mathrm{N}$, destilado.

Solução de $\mathbf{K}_{2} \mathbf{C r}_{2} \mathbf{0}_{7} \mathbf{0 , 1 0 0} \mathbf{N}$. Pesar 4,9035 g de $\mathrm{K}_{2} \mathrm{Cr}_{4} \mathrm{O}_{7}$ p.a, seco a $100-110^{\circ} \mathrm{C}$, dissolver em água destilada, transferir para balão volumétrico de 1 litro e completar o volume.

Solução padronizada de $\mathrm{Na}_{2} \mathbf{S}_{2} \mathbf{0}_{3}$ (aprox. 0,1N). Pesar $25 \mathrm{~g}$ de $\mathrm{Na}_{2} \mathrm{~S}_{2} \mathrm{O}_{3} .5 \mathrm{H}_{2} \mathrm{O}$ p.a , dissolver em água destilada, transferir para balão volumétrico de 1 litro, completar o volume e padronizar.

Solução padronizada de $\mathrm{Na}_{2} \mathbf{S}_{2} \mathbf{O}_{3}$ (aprox. 0,02N). Preparar, no momento do uso, por diluição da solução padronizada aproximadamente $0,1 \mathrm{~N}$.

Solução padrão "estoque" de cobre. Dissolver $0,3930 \mathrm{~g}$ de $\mathrm{CuSO}_{4} .5 \mathrm{H}_{2} \mathrm{O}$ p.a. em água desmineralizada, transferir para balão volumétrico de 1 litro e fazer um volume de aproximadamente 800 $\mathrm{ml}$. Adicionar $5 \mathrm{ml}$ de $\mathrm{H}_{2} \mathrm{SO}_{4}$ concentrado p.a., esperar esfriar e completar o volume. Esta solução contém 100 microgramas de cobre por mililitro.

Soluções padrões de "trabalho" de cobre. Transferir 5,0 - 10,0 20,0 - 30,0 - 40,0 - 50,0 ml da solução estoque para balões volumétricos de $100 \mathrm{ml}$ e completar o volume com água desmineralizada. Estas soluções contém $5,0-10,0-20,0-30,0-40,0$ e 50,0 microgramas de cobre por mililitro.

Solução de dietilditiocarbamato de sódio (DDC - Na) a $1 \%$. Dissolver 1,0 g do sal p.a., tri-hidratado, em água desmineralizada, transferir para balão volumétrico de $100 \mathrm{ml}$ e completar o volume.

Solução de EDTA dissódico a 0,5\%. Dissolver $5,0 \mathrm{~g}$ do sal di- 
hidratado em água desmineralizada, transferir para balão volumétrico de 1 litro e completar o volume.

\section{Método volumétrico}

a - Pesar 2,000 g da amostra bem moída, transferir para frasco de erlenmeyer de $250 \mathrm{ml}$ e adicionar $10 \mathrm{ml}$ de $\mathrm{HNO}_{3}$ e $5 \mathrm{ml}$ de $\mathrm{H}_{2} \mathrm{SO}_{4}$ concentrados.

b - Digerir em chapa aquecedora até o aparecimento de densos fumos brancos.

c) - Esfriar, adicionar $50 \mathrm{ml}$ de água destilada, ferver 1 minuto e esfriar novamente à temperatura ambiente.

d - Adicionar 3 gotas de solução de bromocresol verde a 0,1\% e solução de $\mathrm{NH}_{4} 0 \mathrm{H}$ concentrado até o indicador mudar para cor verde clara $(\mathrm{pH}=4,0)$.

e - Esfriar novamente a temperatura ambiente e, se o indicador mudar para a cor amarela, adicionar mais $\mathrm{NH}_{4} \mathrm{OH}$, gota a gota, até $\mathrm{o}$ indicador tornar-se verde-claro outra vez.

$\mathrm{f}$ - Adicionar $2 \mathrm{~g}$ de $\mathrm{NH}_{4} \mathrm{HF}_{2}$ p.a., agitar e deixar em repouso por 5 minutos.

g - Adicionar $20 \mathrm{ml}$ de solução de KI a $50 \%$, agitar e titular com a solução padronizada de $\mathrm{Na}_{2} \mathrm{~S}_{2} \mathrm{O}_{3}, 0,02 \mathrm{~N}$ até amarelo claro. Adicionar $1 \mathrm{ml}$ da solução de amido a $1 \%$, continuar titulando até o desaparecimento da cor azul e não tornar a escurecer durante 20 segundos. Anotar o volume gasto.

\section{Método colorimétrico}

\section{Preparo da curva padrão}

a - Transferir $1,0 \mathrm{ml}$ de cada solução padrão de trabalho para funis de separação de $125 \mathrm{ml}$.

b - Adicionar $50 \mathrm{ml}$ de solução de EDTA a 0,5\%, 3 gotas de solução de fenolftaleina a $0,1 \%$ e gotas de solução de hidróxido de amonio $(1+1)$ até a viragem do indicador para cor rósea, agitando após a adição de cada solução; em seguida adicionar $1 \mathrm{ml}$ da solução de $\mathrm{DDC}-\mathrm{Na}$ a $1 \%$ e água desmineralizada até obter-se um volume final de aproximadamente $60 \mathrm{ml}$ em cada funil.

c-Adicionar exatamente $10 \mathrm{ml}$ de tetracloreto de carbono p.a. em cada funil e agitar vigorosamente durante 2 minutos.

d - Após a separação das fases, drenar o solvente, através de algodão, para tubos de colorímetro.

e - Efetuar as leituras usando filtro n. ${ }^{0} 42$ do colorímetro Klett - Summerson. 
$\mathrm{f}$ - Desenvolver uma prova em branco.

Com os dados obtidos fazer a curva relacionando as observancias com microgramas de cobre das soluções padrões e ou calcular a equação de regressão da mesma.

\section{Preparo dos extratos de fertilizantes.}

a. Transferir $2,000 \mathrm{~g}$ da amostra bem moída para copo de $150 \mathrm{ml}$.

b. Adicionar $30 \mathrm{ml}$ de solução de $\mathrm{HC} 1(1+1)$ destilado, cobrir com vidro de relógio e ferver lentamente em chapa aquecedora por 15 minutos.

c. Esfriar, adicionar 20-30 $\mathrm{ml}$ de água desmineralizada e filtrar por papel Whatman n. ${ }^{\circ} 1$, recebendo o filtrado num balão de $250 \mathrm{ml}$. Lavar completamente o copo e o resíduo e completar o volume.

\section{Preparo do extrato de fertilizante contendo resíduos organicos}

Apesar de pouco comum, resíduos organicos como tortas, farinhas, etc., podem se fazer presentes em misturas de fertilizantes.

a. Transferir 2,000 g da amostra bem moída para copo de $250 \mathrm{ml}$.

b. Adicionar $10 \mathrm{ml}$ de $\mathrm{HNO}_{3}$ e $5 \mathrm{ml}$ de $\mathrm{H}_{2} \mathrm{SO}_{4}$ concentrados p.a. e digerir em chapa aquecedora até o aparecimento de densos fumos brancos.

c. Retirar da chapa, esperar esfriar e acrescentar mais $1 \mathrm{ml} \mathrm{de}$ $\mathrm{HNO}_{3}$ e $1 \mathrm{ml}$ de $\mathrm{HClO}_{4}$ concentrados, aquecer novamente até o aparecimento de densos fumos brancos e prosseguir o aquecimento até próximo à secura .

d. Esfriar, acrescentar 50-60 ml de água desmineralizada e ferver por 1 minuto.

e. Esfriar e filtrar por papel Whatman n. 1 recebendo o filtrado num balão de $250 \mathrm{ml}$. Lavar completamente o copo e o resíduo e completar o volume.

Observação. Esse processo de preparo do extrato, quando usado para o método volumétrico, é mais conveniente que seja feito em frasco de Erlenmeyer de $250 \mathrm{ml}$ e dispensa-se a filtração.

\section{Determinação}

Transferir 5,0 $\mathrm{ml}$ do extrato de fertilizante para funil de separação de $125 \mathrm{ml}$ e prosseguir conforme descrito a partir do item $\mathbf{b}$ do preparo da curva padrão. Desenvolver uma prova em branco a partir do preparo do extrato. 


\section{RESULTADOS OBTIDOS E DISCUSSÃO}

\section{Influencia do ânion fosfato}

Considerando que os fertilizantes podem conter elevado teor de fosfato, nesses níveis a influencia desse ânion no método colorimétrico do dietilditiocarbamato não está devidamente esclarecida. Com esse objetivo foi desenvolvido um estudo em soluções puras, procedendo-se conforme descrito no preparo da curva padrão, porém, adicionando quantidades variáveis de solução padrão de fostato $\left(\mathrm{K} \mathrm{H}_{2} \mathrm{PO}_{4}\right)$ após a adição dos padrões de cobre. Os resultados obtidos encontram-se no quadro 2.

Quadro 2 - Absorbância de soluções padrões de cobre, em presença de diversas quantidades de fosfato.

\begin{tabular}{|c|c|c|c|c|}
\hline \multirow{2}{*}{$\begin{array}{l}\text { microgramas } \\
\text { de cobre }\end{array}$} & \multicolumn{4}{|c|}{$\mathrm{mg} \mathrm{de} \mathrm{PO}_{4}^{3-}$ adicionados } \\
\hline & 0,0 & 0,4 & 2,0 & 4,0 \\
\hline 10 & 102 & 102 & 102 & 100 \\
\hline 30 & 280 & 280 & 276 & 274 \\
\hline 50 & 430 & 430 & 430 & 430 \\
\hline
\end{tabular}

Os dados revelam que até $4 \mathrm{mg}$ de $\mathrm{PO}_{4}^{3^{-}}$não influem na determinação colorimétrica do cobre pelo método do DDC - Na.

\section{Determinação do cobre nas amostras de fertilizantes}

Em todas as amostras de fertilizantes o teor de cobre foi determinado pelos métodos volumétrico e colorimétrico, estando os resultados descritos no quadro 3.

Quadro 3 - Resultados da determinação do cobre em fertilizantes minerais, pelos métodos volumétrico e colorimétrico. Média de 5 determinacões.

\begin{tabular}{|c|c|c|c|c|c|}
\hline \multirow{2}{*}{\multicolumn{2}{|c|}{ Amostras }} & \multicolumn{2}{|c|}{ Método volumétrico } & \multicolumn{2}{|c|}{ Método colorimétrico } \\
\hline & & $\%$ de $\mathrm{Cu}$ determ. & C.V.\% & $\%$ de $\mathrm{Cu}$ determ. & C.V.\% \\
\hline$A+2 \%$ & $\mathrm{CuSO}_{4} \cdot 5 \mathrm{H}_{2} \mathrm{O}$ & $0,44 \pm 0,013$ & 3,0 & $0,50 \pm 0,007$ & 1,5 \\
\hline$A+4 \%$ & ," & $0.84 \pm 0,021$ & 2,5 & $0,96+0,020$ & 2,1 \\
\hline$A+8 \%$ & $"$ & $1.97 \pm 0,022$ & 1,1 & $1,93 \pm 0,015$ & 0,8 \\
\hline$B+2 \%$ & $"$ & $0,44 \pm 0,009$ & 2,0 & $0,47 \pm 0,017$ & 3,5 \\
\hline$B+4 \%$ & $"$ & $0,89 \pm 0,017$ & 1,9 & $1,00 \pm 0,023$ & 2,2 \\
\hline $\mathrm{B}+8 \%$ & $"$ & $1,83 \pm 0,025$ & 1,4 & $1,93 \pm 0,025$ & 1,3 \\
\hline$C+2 \%$ & $"$ & $0,44 \pm 0,018$ & 4,1 & $0,48 \pm 0,017$ & 3,5 \\
\hline$D+4 \%$ & $"$ & $0,88 \pm 0,029$ & 3,3 & $0,97 \pm 0,099$ & 1,0 \\
\hline$E+8 \%$ & $"$ & $1,83 \pm 0,033$ & 1,8 & $1,90 \pm 0,015$ & 0,8 \\
\hline
\end{tabular}


Esses resultados, comparados estatisticamente através do teste t de significancia (GOMES, 1958 e 1970), possibilitou concluir que os dois métodos empregados não diferem significativamente entre os níveis de $1 \%$ e $5 \%$ de probabilidades. Adotando os valores obtidos pelo método volumétrico como os verdadeiros, o método colorimétrico pode ser considerado suficientemente exato.

Por outro lado, os valores dos coeficientes de variação permitem concluir que o método colorimétrico apresenta precisão adequada, comparável a do método volumétrico. O coeficiente de variação médio apresentado pelo método volumétrico foi de $2,34 \%$ e pelo método colorimétrico foi de $1,86 \%$.

\section{Determinação do cobre em fertilizantes contendo resíduos organicos.}

Para a determinação do cobre e outros nutrientes em fertilizantes contendo resíduos organicos, Mc BRIDE (1967) recomenda a incineração da amostra e posterior extração com $\mathrm{HC1}$, o que, porém, é relativamente demorado.

Inicialmente estudou-se a eficiencia do ataque nitro-sulfúrico, empregado no método volumétrico, na destruição da matéria organica. Assim, da mistura $\mathbf{E}$ com $8 \%$ de $\mathrm{CuSO}_{4} .5 \mathrm{H}_{2} 0$, foram preparadas amostras contendo aproximadamente $0,2,4,6,8$ e $10 \%$ de torta de amendoim. Os teores de cobre determinados volumetricamente estão relatados no quadro 4 .

Quadro 4 - Teores de cobre determinados pelo método volumétrico na mistura $\mathrm{E}$ com $8 \%$ de $\mathrm{CuSO}_{4} \cdot 5 \mathrm{H}_{2} \mathrm{O}$ e contendo diversas quantidades de torta de amendoim.

\begin{tabular}{c|c}
\hline Conteúdo de torta de amendoim & $\%$ de $\mathrm{Cu}$ determinado \\
\hline 0 & 1,84 \\
2 & 1,86 \\
4 & 1,84 \\
6 & 1,62 \\
8 & 1,65 \\
10 & 1,73 \\
\hline
\end{tabular}

Portanto o método volumétrico mostrou-se falho na determinação do cobre em fertilizantes contendo mais de $4 \%$ de resíduos organicos.

Em face desses resultados estudou-se um processo de ataque que servisse tanto ao método colorimétrico como ao volumétrico. Dentre os processos estudados (via seca, água régia, etc.) o mais 
satisfatório foi o nitro-sulfúrico-perclórico. Usando este processo, determinou-se conteúdo de cobre das amostras $\mathrm{C}+2 \%$ de $\mathrm{CuSO}_{4} .5 \mathrm{H}_{2} \mathrm{O}_{4}$ e $\mathrm{E}+8 \%$ de $\mathrm{CuSO}_{4} .5 \mathrm{H}_{2} \mathrm{O}$, contendo diversos teores de torta de amendoim, volumétrica e colorimetricamente, estando os resultados descritos no quadro 5.

Conforme se observa, o processo nitro-sulfúrico-perclórico para o ataque de amostras de fertilizantes contendo resíduos organicos, foi eficiente e se presta tanto para o método colorimétrico como para o método volumétrico.

Quadro 5 - Teores de cobre determinados volumétrica e colorimetricamente, em fertilizantes contendo diversas quantidades de torta de amendoim, usando o ataque nitro-sulfúrico-perclórico.

\begin{tabular}{c|c|c|c}
\hline \multirow{2}{*}{ Amostras } & \multirow{2}{*}{$\begin{array}{c}\% \text { de torta } \\
\text { de } \\
\text { amendoim }\end{array}$} & $\begin{array}{c}\text { Método } \\
\text { volumétrico }\end{array}$ & $\begin{array}{c}\text { Método } \\
\text { colorimétrico }\end{array}$ \\
\cline { 4 - 5 } $\mathrm{C}+2 \% \mathrm{CuSO}_{4} \cdot 5 \mathrm{H}_{2} \mathrm{O}$ & 0 & 0,51 & 0,51 \\
$\mathrm{C}+2 \% \mathrm{CuSO}_{4} \cdot 5 \mathrm{H}_{2} 0$ & 2 & 0,51 & 0,51 \\
$\mathrm{C}+2 \% \mathrm{CuSO}_{4} \cdot 5 \mathrm{H}_{2} \mathrm{O}$ & 5 & 0,50 & 0,50 \\
$\mathrm{C}+2 \% \mathrm{CuSO}_{4} \cdot 5 \mathrm{H}_{2} \mathrm{O}$ & 10 & 0,51 & 0,50 \\
$\mathrm{E}+8 \% \mathrm{CuSO}_{4} \cdot 5 \mathrm{H}_{2} \mathrm{O}$ & 0 & 1,99 & 1,97 \\
$\mathrm{E}+8 \% \mathrm{CuSO}_{4} \cdot 5 \mathrm{H}_{2} \mathrm{O}$ & 2 & 1,94 & 2,00 \\
$\mathrm{E}+8 \% \mathrm{CuSO}_{4} \cdot 5 \mathrm{H}_{2} \mathrm{O}$ & 5 & 1,99 & 1,91 \\
$\mathrm{E}+8 \% \mathrm{CuSO}_{4} \cdot 5 \mathrm{H}_{2} \mathrm{O}$ & 10 & 1,99 & 1,94 \\
\hline
\end{tabular}

\section{CONCLUSÕES}

Os resultados obtidos permitiram as seguintes conclusões.

a) o método colorimétrico do dietilditiocarbamato de sódio não é influenciado por uma quantidade de fosfato de até $4 \mathrm{mg}$.

b) O cobre de fertilizantes minerais é extraído por simples fervura da amostra com solução de $\mathrm{HC1}(1+1)$ por 15 minutos.

c) O ataque nitro-sulfúrico não foi adequado para amostras de fertilizantes contendo mais de $4 \%$ de resíduos organicos, quer para o método volumétrico como para o colorimétrico. Por outro lado o ataque nitro-sulfúrico-perclórico foi eficaz para ambos os métodos.

d) o método colorimétrico do dietilditiocarbamato de sódio mostrou possuir precisão e exatidão satisfatórias para a determinação do cobre em fertilizantes, associadas a sua simplicidade e sensibilidade. 


\section{SUMMARY}

\section{Colorimetric Determination of Copper in Fertilizers.}

This paper deals on the adaptation of the diethildithiocarbamate colorimetric method in the determination of copper in fertilizers. Were studied the influence of high phosphate concentrations, preparation techniques of extracts of mineral fertilizers and fertilizers containing organic materials and precision and accuray of the method comparatively with the iodometric method.

The results allowed to conclude that the diethildithiocarbamate colorimetric method is not influenced by amounts of phosphate up to $4 \mathrm{mg}$. Its precision and accuray for copper determination in fertilizers is comparable to the iodometric method.

\section{LITERATURA CITADA}

CHENG, K.L. \& R.H. BRAY, 1953, Two specifics methods of determining copper in soil and in plant material. Anal. Chem., 25 (4): 655-659.

FISKELL, J. G. A., 1965. Copper. Em: Methods of Soil Analysis. Part 2. p. 1078-1089, n. 9 in the serie Agronomy. Ed. por C. A. Black e outros. American Society of Agronomy, Inc. Publisher. Medison, Wisconsin, USA.

GOMES, F. P., 1968. Iniciação à Estatística. 2. edição. Livraria Nobel S. A., $207 \mathrm{pp}$.

GOMES, F. P. 1970. Curso de Estatística Experimental. 4. ${ }^{a}$ edição. Livraria Nobel S. A. $430 \mathrm{pp}$.

JACINTHO, A.O., 1967. A determinação do cobre pelo método colorimétrico do dietilditiocarbamato de sódio. Tese apresentada à E.S.A. "Luiz de Queiroz" para obtenção do título de "Doutor em Agronomia" 82 pp. mimeografadas.

JACKSON, M. L., 1958. Soil Chemical Analysis. Prentice - Hall, Inc., Englewood Criffs, New Jersey. $498 \mathrm{pp}$.

Mc BRIDE, C. H., 1967. Determination of secondary and minor plant nutrients in fertilizers by atomic absorption spectrophotometry: third collaborative study. J. Ass. Off. Anal. Chem. 50 (2): 401-407.

NELSON, W. L. \& G. L. TERMAN, 1963. Nature, Behavior, and Use of Multinutrient (mixed) Fertilizers. Em: Fertilizers, Technology and Usage, Ed. by M. H. Mc Vickar, G. L. Bridger and L. B. Nelson. Soil Science Society of America. Wisconsin. pp 379-427.

OFFICIAL METHODS OF ANALYSIS OF THE A. O. A. C., 1965. 10th ed., pp. 23-24 e 29-30. Published by Ass. of Off. Agr. Chem., Washington, D.C.

SANDELL, E. B. 1959. Colorimetric Determination of Traces of Metals. 3rd ed. Interscience Publishers. Inc., New York. $1032 \mathrm{pp}$. 
